DARK RESONANCE

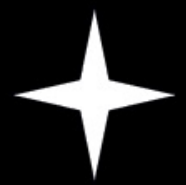

\title{
Sensitive magnetometers based on dark states
}

\author{
I Luigi Moi ${ }^{1}$, Stefka Cartaleva ${ }^{2}$ - DOI: 10.1051/epn/2012603 \\ n 1Physics Department - University of Siena - Italy - moi@unisi.it \\ - 2Institute of Electronics - Bulgarian Academy of Sciences - Sofia, Bulgaria
}

\section{So-called Dark States provide another example of fundamental research which yields unexpected applications. Here is an all-optical magnetometer that can detect tiny fields even in an unshielded environment. As an obvious application a human magneto-cardiogram (MCG) has been recorded. And there are more promising applications.}

$\Delta$ The first observation of dark resonances (marked by a star) in dye-laserinduced fluorescence of $\mathrm{Na}$ atoms, caused

by a spatially

inhomogeneous magnetic field along the laser beam.

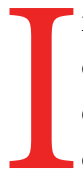
$\mathrm{n}$ recent years interest has been growing into the fascinating properties of dark resonances. Most of the experiments have been carried out in alkali atoms due to their favourable level structure and the commercially available diode lasers, resulting in numerous applications. Teams from Siena and Sofia developed an all-optical magnetometer based on such dark resonances. The dark-state effect was discovered in 1976 by G. Alzetta, A. Gozzini, L. Moi and G. Orriols [1], while they were working on optical-pumping experiments with a multimode dye laser. They wanted to make the observation of multi-photon radio-frequency resonances in sodium atoms easy, i.e., observable by the naked eye. To this end, they introduced a magnetic field (MF) that was constant in time but spatially inhomogeneous along the laser beam, so as to produce Zeeman splitting of groundstate hyperfine levels (Fig.1a, left). In this way, an applied radio-frequency field was resonant only if it matched the Zeeman splitting. As a result, a bright radio-frequency resonance appeared (Fig.1a, right).

During these experiments, besides the bright resonances, a new "dark" resonance was observed, which does not require any radio-frequency field. In the introductory illustration only the dark resonance is shown. The new dark state (DS) effect appears in a position along the laser beam where the frequency difference $\left(\omega_{10}-\omega_{20}\right)$ between two optical frequencies is equal to the frequency splitting $\omega_{12}$ between two Zeeman sublevels (Fig.1b, left). This is the resonant condition for the stimulated Raman transitions between the two longliving ground levels. Theoretical studies described the dark resonance as a coherent superposition of two long-living ground levels prepared by the bi-chromatic laser field, and 'atomic population trapping' was introduced, leading to the term Coherent Population Trapping (CPT) [2]. 
A striking property of the DS is that its spectral width is only 10 to $100 \mathrm{~Hz}$ (see Fig.1b, right). This is because it is determined mainly by the long lifetime of the two ground levels, so the much larger spectral width of the excited level is not involved. Thus, the CPT resonances can be extremely narrow features allowing performances both at the level of basic physics experiments and of practical applications. Recently a complete suppression of fluorescence in narrow resonance has been realized exciting all ground-state Zeeman sublevels of sodium [3].

Different approaches have been adopted to observe CPT resonance. Most frequently Zeeman sublevels of the two hyperfine ground levels (with $\omega_{12} \sim 2 \div 9 \mathrm{GHz}$ for different alkali atoms) are coupled by two coherent optical fields [4], commonly obtained by diode laser frequency modulation around $\omega_{12}$ that requires relatively high modulation power. To reduce this problem, we proposed $[5,6]$ to shift laser modulation to the $\mathrm{kHz}$ region by coupling Zeeman sublevels of the same ground-state hyperfine level, where $\omega_{12}$ is a few hundred $\mathrm{kHz}$ in earth and lab MFs. In addition, the involvement of a single hyperfine transition in the CPT preparation leads to observation of a new narrow resonance of enhanced absorption [6,7]. The recently expanding interest in the topic is due not only to the fascinating physics involving quantum coherence but also to the fact that there are many potential applications with relevance for fundamental studies, such as slowing of light, STIRAP, velocity-selective $\mathrm{CPT}$ and quantum information storage, as well as for development of new techniques/devices like atomic clocks/magnetometers.

Here we describe the development of an all-optical magnetometer based on the DS, which is promising for realization of low-cost and easily-operated practical devices for weak MF measurement.

\section{Scheme for magnetic field (MF) measurements}

In the developed approach (Fig. 2), bi-chromatic/polychromatic coherent optical fields couple Zeeman sublevels within a single hyperfine level, resulting in observation of DS resonance with $\Gamma<100 \mathrm{~Hz}$, in a magnetically unshielded but partially compensated environment. The CPT resonance observed as a function of the modulation frequency makes absolute measurement of the MF value possible. Constant earth and lab MFs shift the resonance center at $\omega_{\text {mod }}=\mathrm{B} / \mathrm{k}$ without causing its broadening. The first step of the measurement is to find this point and to determine

V FIG. 1: (a) Formation of bright optical pumping resonance. (left) Due to fluorescence decay, a light field of frequency $\omega_{10}$, resonant with the 1-0 transition, produces optical pumping (accumulation) of atoms in the ground level 2, non-interacting with the light. Consequently, the population in level 1 decreases. (right) As the splitting ( $\omega_{12}$ ) between levels 1 and 2 is determined by the applied magnetic field, the radio-frequency field (with $\omega_{\mathrm{RF}}$ ) will couple the ground levels if $\omega_{\mathrm{RF}}=\omega_{12}$, repopulating level 1 and producing enhanced absorption and a bright resonance (enhanced fluorescence). (b) Dark-state resonance formation: (left) basic scheme for Coherent Population Trapping (CPT) - only two optical fields are used, i.e., no radio-frequency field is involved in the CPT preparation; (right) when the two optical fields $\left(\omega_{10}\right.$ and $\left.\omega_{20}\right)$ are components of a modulated laser field separated by modulation frequency $\omega \bmod \left(=\omega_{10}-\omega_{20}\right)$, the dark resonance is centered at the modulation frequency determined by the ground level splitting $\omega_{12}=\omega_{10}-\omega_{20}$.

Excited level: 0

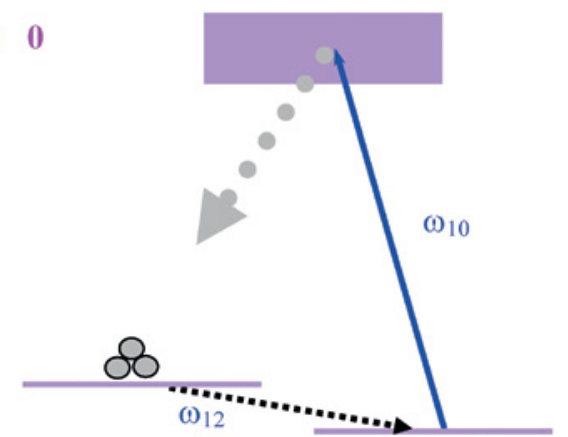

1

Long living ground levels: 1 and 2

Excited level: 0

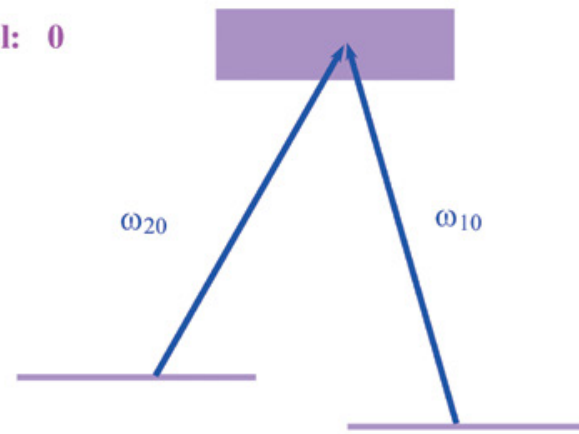

(a)

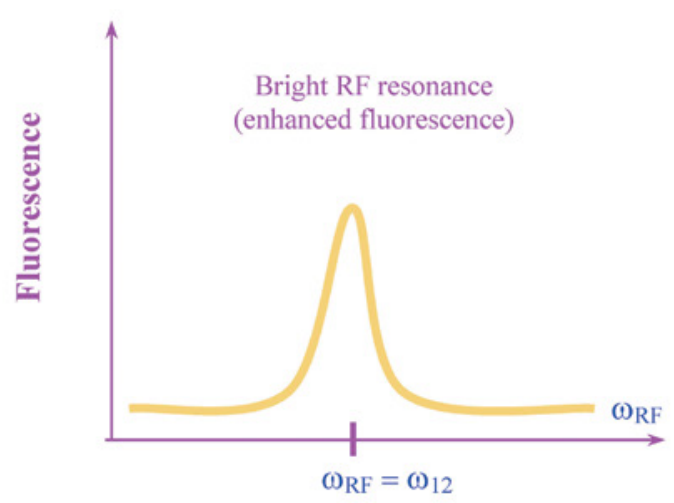

(b)

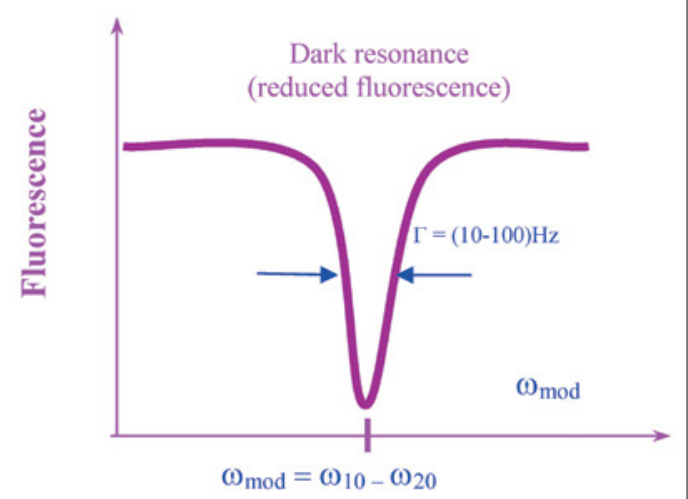


B. At the second step, the modulation frequency $\omega_{\bmod }$ is fixed and the small magnetic field to be measured (in the interval $\Delta \mathrm{B}=\mathrm{k} \Gamma$ ) is switched on. Thus, the resonance profile enables precise measurement of small MF fluctuations

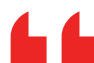

This MCG measurement is passive (no radiation imposed to subject), completely noninvasive and contact-free. in time, superimposed on a large magnetic background. In this way, the requirements for earth and lab MF shielding are strongly relaxed, which is a significant advantage because the shielding is about an order of magnitude more expensive than the magnetometer. Note that other techniques performing the most precise measurement of MF fluctuations around zero value require expensive MF shielding.

\section{First human magneto-cardiogram (MCG) in unshielded environment}

In 2007, an encouraging result was obtained [8] with an experimental apparatus based on CPT and aimed at detecting a MCG in a magnetically compensated but unshielded surrounding. The system was suitable to compensate for some dc components of the environmental MF and the first order MF inhomogeneities, thus reducing the resonance line width to few tens of $\mathrm{Hz}$. Environmental noise was cancelled by making use of a differential measurement. Synchronous data acquisition with a reference signal (electro-cardiogram/pulse-meter) improved the signal-to-noise ratio by off-line averaging. The setup has the significant advantages of working at room temperature with a smallsize magnetic sensor, and the possibility of fast adjustments of the dc bias MF, which makes the sensor suitable for detecting a biomagnetic signal of any orientation and in any position on the patient's chest.

This all-optical technique does not require coils in the proximity of the sensor cell to produce direct radiofrequency magnetic excitation. The sensor consists of a glass cell containing alkali vapor, and the laser light is brought to and from the cell by optical fibers.

The potential of the magnetometer for magnetocardiographic applications in unshielded environments is illustrated by Fig. 3. Multiple MCG traces are averaged, in order to improve the signal-to-noise. The signals are filtered with a single low-pass filter and the deterministic noises (e.g., originating from the main supply and its harmonics) are subtracted off-line.

The simplicity of the method, its low cost, and the low cost of maintenance may be crucial factors for its dissemination in clinical applications. Note that this MCG measurement is passive (no radiation imposed to subject), completely non-invasive and contact-free.

\section{Other promising applications}

A dual-channel self-oscillating approach has been developed, based on a wide frequency modulation of the pump laser and registration of the nonlinear magneto-optical rotation of the probe laser [9]. The self-oscillating operation makes the magnetometer insensitive to the slow drifts of the MF (of several nano-Tesla), thus making possible long time records up to the duration of the working days. The sensor was used for the nuclear magnetic resonance (NMR) registration, detecting both the dc magnetization and the nuclear spins precession of remotely polarized

v FIG. 2: Principle scheme of an all-optical DS magnetometer. The modulated light (with components separated by $\omega_{\text {mod }}$ ) is effectively produced by direct modulation of a diode laser current. The measured magnetic field B splits levels 1 and 2 of alkali atoms (Fig.1) at frequency $\omega_{12}$ related to the MF by $B=k \omega_{12}$, where $k$ is determined by atomic constants. In unshielded and partially-compensated environments, the light produces a dark resonance, centered at $\omega_{12}=91228 \mathrm{~Hz}$ (denoted by asterisk), which is the modulation frequency ( $\omega$ mod) of the laser. The dark-resonance spectral width is $\Gamma \sim 90 \mathrm{~Hz}$; its first derivative is shown by curve (a). The resonance corresponds to a $M F$ variation $\Delta B=k \Gamma$. If the modulation frequency is kept constant ( $\omega_{\text {mod }}=\omega_{12}=$ constant), the obtained linear dependence $C D$ (within the resonance profile) allows measuring MF fluctuations less than $\triangle B$, based on the registration of the transmitted light intensity with time - shown in the curve on the right (b). In unshielded and partially-compensated environments the value $\Delta B$ is less than $B$ by several orders of magnitude.

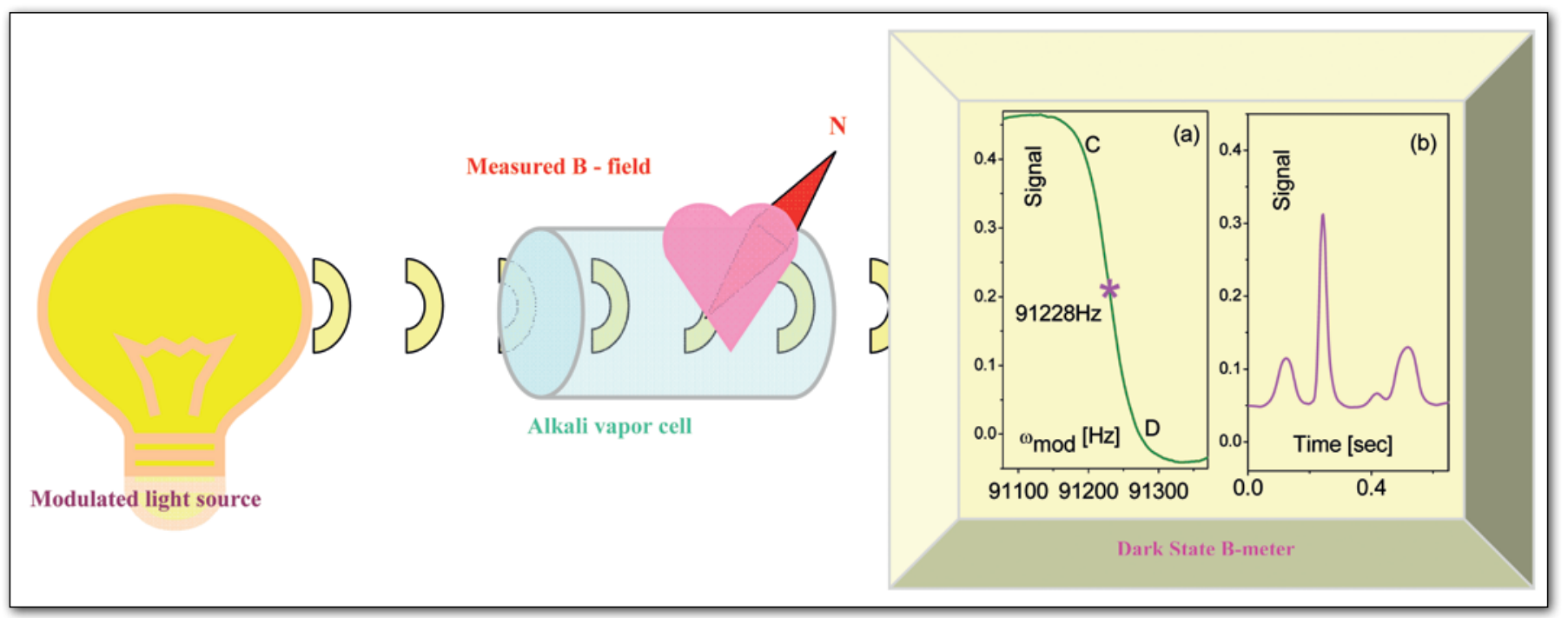


hydrogen nuclei in water samples. The optical magnetometer reaches a sensitivity of $100 \mathrm{fT} . \mathrm{Hz}^{-1 / 2}$ worsened to $2 \mathrm{pT}$. $\mathrm{Hz}^{-1 / 2}$ due to MF gradients in an unshielded environment. To facilitate averaging of bias-MF-dependent signals and to improve the signal/noise ratio in unshielded environment, an automatic system has been developed [10] for active compensation of time-dependent earth and urban MFs using the dual channel scalar magnetometer. The system made possible to stabilize the bias field within a bandwidth of several tens of $\mathrm{Hz}$, counteracting MF fluctuation with attenuation levels exceeding $40 \mathrm{~dB}$.

Developed optical magnetometers have been successfully tested for monitoring of earth MF variations, showing that the approach is quite robust against urban electromagnetic noise [11].

A lot of effort has been put by US scientists for the development of practical chip-scale atomic clock and magnetometer, based on the DS. More recently a special care has been taken to avoid electrical connections to the sensor, i.e. making the sensor head largely non-magnetic and its performance free of internal MFs [12].

\section{Outlook}

The Siena group, lead by V. Biancalana, is presently involved in a project for the magnetic characterization of magneto-nano-particle hydrogels. Further campaigns are foreseen in MCG as well as in ultra-low-field NMR, including collaboration with researchers involved in developing unconventional techniques for nuclear prepolarization. The Sofia team is involved in studies of miniaturized optical magnetometer based on DS and in the improvement of its signal/noise.

\section{Conclusion}

The DS magnetometer is a versatile device offering the possibility to build up non-magnetic sensors: no internal MFs perturb the field to be measured. This is an important issue when multichannel sensors are developed. Absence of coils minimizes the size and optical fibers may easily address each single detection point. It can be used also for remote control or work in hostile environment.

\section{Acknowledgements}

Both authors are strongly indebted with the colleagues from Pisa, Siena and Sofia for their fundamental contributions to the studies of the dark/bright state phenomena. Many thanks to G. Alzetta, A. Gozzini great scientists and teachers in optics, as well as to S. Gozzini, F. Renzoni, E. Arimondo, V. Biancalana, Y. Dancheva, J. Belfi, G. Bevilacqua, E. Mariotti, C. Marinelli, E. Breschi, S. Gateva, E. Alipieva, G. Todorov, D. Slavov, C. Andreeva, T. Karaulanov, E. Taskova for fruitful and nice collaborations. The authors acknowledge financial support from the CNSIM, Monte dei Paschi di Siena, and the Bulgarian NCSR (grant No: DO 02-108/22.05.2009).

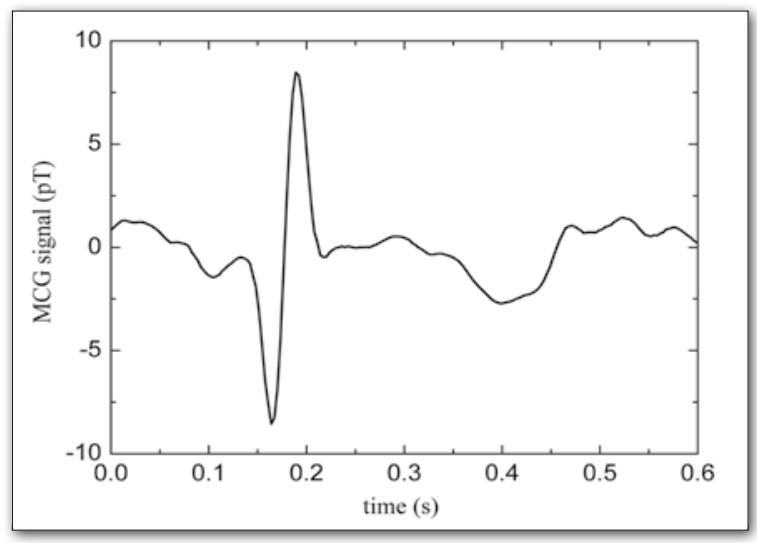

4 FIG. 3: MCG signal (83 averages) of an 8 year old boy recorded in an unshielded environment. Note that signals of order $10^{-12}$ tesla are detected. This result is kindly provided by V. Biancalana (unpublished).

\section{References}

[1] G. Alzetta, A. Gozzini, L. Moi, G. Orriols, Nuovo Cimento 36, 5 (1976).

[2] E. Arimondo, Prog.Opt. 35, 257 (1996).

[3] G. Alzetta, S. Gozzini, A. Luchesini, S. Cartaleva, T. Karaulanov, C. Marinelli, L. Moi, Phys. Rev. A 69, 063815 (2004).

[4] M. Stahler, S. Knappe, C. Affolderbach, W. Kemp, R. Wynands, Europhys.Lett. 54, 323 (2001).

[5] V. Biancalana, Y. Dancheva, E. Mariotti, L. Moi, S. Cartaleva, C. Andreeva, European Patent No EP1570282, Priority 03/12/2002.

[6] C. Andreeva, G. Bevilacqua, V. Biancalana, S. Cartaleva, Y. Dancheva, T. Karaulanov, C. Marinelli, E. Mariotti, L., Appl. Phys. $B$ 76, 667 (2003),

[7. Y.Dancheva, G.Alzetta, S.Cartaleva, M.Taslakov, Ch.Andreeva, Opt. Commun. 178 (2000) 103; F. Renzoni, S. Cartaleva, G. Alzetta, E. Arimondo, Phys.Rev. A 63, 065401 (2001).

[8] J. Belfi, G. Bevilacqua, V. Biancalana, S. Cartaleva, Y. Dancheva, L. Moi, JOSA B 24, 2357 (2007).

[9] J. Belfi, G. Bevilacqua, V. Biancalana, S. Cartaleva, Y. Dancheva, K. Khanbekyan, L. Moi, JOSA B 26, 910 (2009).

[10] J. Belfi, G. Bevilacqua, V. Biancalana, R. Cecchi, Y. Dancheva, and L. Moi, Rev. Sci. Instrum. 81, 065103 (2010).

[11] S. Cartaleva, S. Gateva, E. Alipieva, A. Yanev, G. Todorov, D. Slavov, C. Andreeva, T. Karaulanov, E. Taskova, L. Petrov, V.Sarova, N. Petrov, Annual report 2004, p.109: www.ie-bas.dir.bg/Presentation.htm

[12] P. D. D. Schwindt, S. Knappe, V. Shah, L. Hollberg, J. Kitching, L.-A. Liew, et al., Appl. Phys. Lett. 85, 6409 (2004) ; J. Preusser, S. Knappe, J. Kitching, V. Gerginov, 2009 IEEE International Frequency Control Symposium Joint with the $22^{\text {nd }}$ European Frequency and Time Forum , art. no. 5168385, p. 1180. 